\title{
Transnacionalización, integración social y diversidades de capitalismo en el este y en el sur*
}

\author{
Lászlo Bruszt** \\ Béla Greskovits***
}

\begin{abstract}
Resumen
El presente artículo analiza los modos de integración internacional, los perfiles de inserción global, la presencia o ausencia de capacidades transformativas de los Estados y la cohesión social como dimensiones que interactúan en la definición de diversidades de capitalismo en la Europa del Este postsocialista y en América Latina. Sobre esta base, se sostiene que el paradigma de desarrollo dependiente de Cardoso y Faletto mantiene su validez. En efecto, una vez adaptada a las condiciones contemporáneas, la perspectiva de Cardoso y Faletto es capaz de capturar las similitudes y divergencias del desarrollo capitalista en ambas regiones. Los patrones recientes de desarrollo demuestran que, a pesar de que la dependencia -derivada de la distribución desigual de recursos, roles y oportunidadescontinúa siendo un aspecto importante de la división internacional del trabajo, ella ha dejado de ser un factor en contradicción con formas complejas de industrialización. Del mismo modo, a pesar de las relaciones asimétricas que caracterizan a los nuevos regímenes transnacionales de integración, estos permiten e incluso ayudan a la formación de nuevos Estados-naciones con mejores capacidades regulatorias. Finalmente, la dependencia no necesariamente socava la integración social. Más bien, es responsabilidad de la competencia democrática generar un equilibrio entre los requerimientos de una integración que sea internacionalmente promisoria y socialmente aceptable.
\end{abstract}

Palabras clave: Transnacionalización - integración social - diversidad de capitalismo - desarrollo capitalista - mercado mundial - democratización.

\begin{abstract}
This article analyzes the deep and shallow modes of international integration, semicore, semiperipheral, and peripheral profiles of global insertion, and the presence and absence of transformative state capacity and social cohesion, as interacting dimensions of capitalist diversity in post-socialist Eastern Europe and Latin America. On these grounds, it is argued that Cardoso and Faletto's dependent development paradigm maintains validity. When adapted to the new conditions, their approach is able to capture the overlapping and divergent aspects of capitalist development in both regions. Recent patterns of development demonstrate that although dependency, stemming from the unequal distribution of resources, roles, and opportunities, continues to be an important aspect of the international division of labor, it ceases to contradict even complex forms of industrialization. Similarly, notwithstanding the asymmetrical power relationships characterizing them, the new transnational integration regimes allow and sometimes help formation of new nation states with improved regulatory capacities. Finally, dependency does not necessarily undermine domestic social inclusion. Rather, it is up to the democratic competition to strike a balance between the requirements of promising international and socially acceptable domestic integration.
\end{abstract}

Keywords: Transnationalization - social integration - capitalist diversity - capitalist development - world market - democratization.

* Traducción de Aldo Madariaga y Stefano Palestini.

** Departamento de Ciencias Sociales y Políticas, Instituto Universitario Europeo, Florencia, Italia. Correo electrónico: laszlo.bruszt@eui.eu

*** Departamento de Relaciones Internacionales y Estudios Europeos, Universidad Central Europea, Budapest, Hungría. Correo electrónico: greskovi@ceu.hu 


\section{INTRODUCCIÓN}

El estudio comparativo del desarrollo capitalista fuera del ámbito de los países más desarrollados nació cuando los estudiosos de la "periferia" abandonaron la idea de que un solo conjunto de restricciones impuestas externamente determinaba la distribución desigual de recursos, roles y oportunidades en el mercado mundial. El rechazo de esta versión imperialista de la teoría de la dependencia inauguró una nueva forma de evaluar el rol jugado por un conjunto mucho más diverso de factores internos y externos, políticos, económicos y sociales que ayudan o impiden la salida de la periferia y que colocan a los países en diversas trayectorias de desarrollo.

Dependencia y desarrollo en América Latina (Cardoso y Faletto, [1967] 1979), en este sentido, fue el precursor del estudio de las diversidades de capitalismo fuera del centro noroccidental. Cardoso y Faletto exploraron diferentes situaciones de desarrollo capitalista dependiente como conjuntos de límites estructurales y condiciones posibilitadores del desarrollo, argumentando que ciertas configuraciones permitían el desarrollo mientras otras lo dificultaban o colocaban a los países en trayectorias menos promisorias. La pregunta acerca de la "variabilidad de las formas de integración en el mercado mundial", como ha sido formulada por Cardoso en su ensayo escrito cuarenta años después (Cardoso, 2009), permitió la identificación tanto de límites específicos como de estrategias alternativas de desarrollo. En vez de buscar recetas universales, su enfoque se centró en los factores que mantenían a los países en trayectorias de desarrollo separadas. El estudio de dichas trayectorias fue la clave para aprender acerca del espacio existente para los agentes del desarrollo interno, y articular así estrategias que permitieran cambiar situaciones concretas de dependencia. En otras palabras, utilizando un concepto (re)inventado casi dos décadas más tarde por los estudiosos del capitalismo comparado en los países centrales: el desarrollo era path dependent (y no era exclusivamente una función de la relación con el centro) (véase Cardoso, 2009).

En este ensayo nos proponemos construir puentes entre el trabajo de Cardoso y Faletto y nuestros propios estudios comparativos previos con el fin de abordar y capturar de qué manera funciona el capitalismo global, y cómo emerge la diversidad inter e intrarregional dentro de la unidad sistémica tanto en la Europa del Este post-socialista como en Latinoamérica. Para iniciar nuestro análisis, demostraremos en primer lugar que el paradigma del desarrollo dependiente, la búsqueda de combinaciones estructuradas de condiciones limitantes y posibilitadoras, mantiene validez. Dicho paradigma es capaz de generar nuevas proposiciones plausibles sobre la economía política de ambas regiones. Es particularmente útil para desafiar tanto las generalizaciones optimistas y pesimistas sobre los límites y posibilidades del desarrollo, las cuales atribuyen un impacto homogeneizante a los legados del socialismo de Estado, la dependencia de los "capitalismos pobres" o la globalización.

Para fundamentar esta tesis, investigamos cuatro dimensiones claves: los modos de integración internacional; los perfiles de inserción global; los niveles de capacidades transformativas del Estado; y la integración social. Tomamos de Cardoso y Faletto y del debate generado por su trabajo que los roles y oportunidades a lo largo de esas dimensiones pueden interactuar para producir trayectorias de desarrollo tardío variadas y más o menos promisorias en la 
economía global. A la luz de dichas configuraciones, las variedades de capitalismo de la Europa del Este así como de América Latina coinciden en aspectos importantes, tales como la especialización en la división internacional de trabajo, y el grado en general amplio aunque variado de capitalismo extranjero y de liderazgo del Estado en tales roles. Al mismo tiempo, las divergencias interregionales prevalecen tanto en la frecuencia de variedades particulares, así como en algunas de las dimensiones relacionadas tales como las capacidades del Estado para fomentar el desarrollo y reducir las desigualdades, o los beneficios de la integración regional.

Sobre esta base sugerimos un mapa tentativo de la evolución de los conglomerados de capitalismo transnacional y rastreamos su emergencia a través de la interrelación de factores internos y externos. Un análisis sólido de las trayectorias alternativas va más allá de las posibilidades de nuestro ensayo. En su lugar, intentamos bosquejar algunos de los espacios conceptuales en los cuales es posible plantearse preguntas relevantes acerca de la diversidad del desarrollo tardío. De esta manera esperamos poder señalar nuevas posibilidades para comparaciones inter e intrarregionales y así brindar oportunidades para que los especialistas en un área geográfica puedan aprender más acerca de su propio campo a través del conocimiento de experiencias alternativas.

\section{DEPENDENCIA, DESARROLLOY DIVERSIDADES DE CAPITALISMO EN LA ECONOMÍA GLOBAL}

¿Es la dependencia, enraizada en la distribución desigual de recursos, roles y oportunidades, todavía un factor influyente en la división internacional del trabajo? ¿La desigualdad internacional persiste o está en declive? Estas son, claramente, preguntas complejas, y tal como el apasionado debate actual lo atestigua, no se ha podido aún dirimir si "el despegue del resto" ha efectivamente desafiado o no a Occidente (Amsden, 2001; véanse las contribuciones en Studies in Comparative International Development, 38 (1), 2003, pp. 3-42).

Es interesante, sin embargo, que mientras pocos negarían la importancia del contexto y los factores internacionales para las posibilidades y obstáculos que encuentran los países de desarrollo tardío, algunos especialistas se han negado recientemente a analizar la integración económica global en términos de dependencia. Más bien, como ha notado Stallings, "(L)as viejas ideas acerca de la influencia externa han sido abandonadas demasiado rápidamente. Irónicamente, justo cuando las variables internacionales comenzaron a ser especialmente importantes en la década de 1980, éstas desaparecieron de las teorías del desarrollo como factores claves. Como habitualmente sucede, la teoría está desfasada de la realidad" (1992: 43).

Sin embargo, ¿no ha sido precisamente el caso que la globalización ha alterado fundamentalmente la manera en la cual las desigualdades internacionales debieran ser estudiadas? Más concretamente, ¿de qué modo las nuevas dinámicas del capitalismo mundial han afectado la validez y el poder explicativo de la "metodología para el análisis de situaciones concretas de dependencia" de Cardoso y Faletto? (Palma, 1978: 909). Las teorías alternativas contemporáneas, ¿no están mejor preparadas para abordar los problemas y tensiones del capitalismo tardío? 
Nuestra respuesta breve a estas preguntas es que las proposiciones claves de Cardoso y Faletto mantienen validez. Muchos de los aspectos internacionales y domésticos cambiantes de la expansión económica pueden ser integrados en su marco teórico, empleándolo como un útil set de herramientas de investigación. Es por esto que en nuestra visión su paradigma puede ser fructíferamente adaptado a los análisis comparativos de contextos de desarrollo actual espacial e históricamente distintos como los de Europa del Este y Latinoamérica. Resumamos los cambios económicos y políticos más relevantes, así como las maneras en las cuales los especialistas inspirados por el enfoque de Cardoso y Faletto han abordado su complejidad.

La primera piedra angular del paradigma del desarrollo dependiente es que los países se embarcan en trayectorias insertas en los contextos cambiantes de la economía mundial capitalista, cuyas formas históricas implican variados riesgos y oportunidades. La esencia de la dependencia en los países menos avanzados es que ellos constituyen partes diversas al interior de una unidad que es transformada constantemente por las dinámicas de los centros capitalistas. Tal como lo exponen Cardoso y Faletto, "Serán distintos el modo y las posibilidades de desarrollo de una nación que se vincula al sector exportador internacional (...) según se verifique en el periodo del capitalismo predominantemente competitivo o en el periodo predominantemente monopólico. En el primer caso, las oportunidades son más favorables para que los locales encuentren un lugar en el mercado. En el segundo, los monopolios internacionales usualmente intentan controlar la producción local" (1979 [1967]: 25).

La principal transformación de la economía mundial en la década de 1960 consistió en que las inversiones realizadas por las corporaciones transnacionales "han sido dirigidas crecientemente hacia la industria manufacturera en la periferia, la lucha por la industrialización... ha llegado a ser crecientemente el objetivo del capital extranjero. Por esto, dependencia e industrialización dejan de ser contradictorios" (Palma, 1978: 909).

Un aspecto relacionado del capitalismo contemporáneo que Ilama poderosamente la atención es que la (re)industrialización conducida externamente de las áreas menos avanzadas no se ha interrumpido desde entonces, sino que se ha ampliado espacialmente y se ha profundizado funcionalmente. Las corporaciones transnacionales han incorporado un creciente número de economías menos avanzadas en sus cadenas globales de valor y sistemas productivos globales (Gereffi, 1995). En efecto, a través de la reubicación productiva, un puñado de países de desarrollo tardío ha llegado a ser productor y exportador importante de bienes manufacturados complejos, hasta el momento vistos como la principal fuente de las ventajas comparativas de las economías avanzadas por su dependencia de capital físico y humano. Esta nueva ola de expansión de manufacturas complejas ha transformado la naturaleza de la dependencia. Como discutiremos más adelante, para los países en situación de dependencia la distribución desigual de roles y oportunidades aparece bajo nuevas formas e inextricablemente vinculada al liderazgo del capital extranjero.

En nuestros casos específicos, el colapso del sistema socialista condujo a la llegada de un gran número de recién llegados con economías menos avanzadas a la economía global. Mientras buscan nuevos roles en la división global del trabajo, las ex economías socialistas y 
latinoamericanas permanecen dependientes, pero dicha dependencia se desarrolla bajo nuevas formas. Aun cuando dichos países sean relativamente industrializados y ricos en materias primas y/o trabajo (aunque en grados diversos), continúan dependiendo de las economías avanzadas y de las corporaciones transnacionales para satisfacer sus requerimientos de nuevas tecnologías y de capacidades empresariales globales, tales como diseño, abastecimiento de insumos, marketing y gestión del conocimiento. Sin embargo, esta no es la única diferencia entre la economía mundial contemporánea y sus fases anteriores analizadas por Cardoso y Faletto.

Otro cambio importante es que, de modo similar a las corporaciones transnacionales manufactureras, los Estados centrales aparecen también más dispuestos a integrar a sus semiperiferias hoy en día de cuanto lo eran antes. La Unión Europea (UE) y el Acuerdo Norteamericano de Libre Comercio (NAFTA), en ese orden, son casos para mencionar. Luego del nuevo milenio, debido a una variedad de razones políticas, económicas y de seguridad, los países del Este-Central Europeo (ECE) han continuado sus esfuerzos por alcanzar los estándares de los países más avanzados del norte y oeste de Europa desde el interior de la UE, su más avanzada e integrada área económica y política. En una escala considerablemente más modesta, el NAFTA también pareciera anunciar una nueva época de la integración norte-sur. En consecuencia, el impacto de diversos regímenes transnacionales de integración (RTI) ${ }^{1}$ debe ser incorporado en la conceptualización del desarrollo tardío, especialmente desde que la UE y su proceso de integración y ampliación han jugado un rol fundamental en los países del ECE (Bruszt y McDermott, 2009).

La UE es similar a otros acuerdos de comercio regional que han extendido mercados por medio de una distribución negociada de beneficios y oportunidades entre miembros caracterizados típicamente por relaciones de poder altamente asimétricas. Del mismo modo, el impacto de la UE sobre los Estados miembros y las instituciones regulativas domésticas difiere respecto a otros regímenes de integración tales como el NAFTA. La UE impone una condicionalidad extensa sobre los miembros aspirantes de manera de mejorar sus capacidades estatales y de dirigir el cambio institucional hacia los estándares típicos del centro formado por la Europa Occidental. Yendo más allá de la condicionalidad, la UE también emplea programas de asistencia ayudando a los actores públicos, privados y de la sociedad civil en la construcción de instituciones regulativas efectivas en campos relacionados con el desarrollo. Por último, en el proceso de acceso, la UE ha fortalecido la cooperación transfronteriza entre actores domésticos variados y sus redes transnacionales orientadas al monitoreo del cumplimiento de la condicionalidad impuesta a los nuevos miembros.

De lo anterior derivamos una proposición en línea con el paradigma del desarrollo dependiente: dependencia y fortalecimiento de las capacidades estatales dejan de ser

$1 \quad \mathrm{~N}$. del T.: El concepto de regímenes transnacionales de integración refiere a conjuntos de principios, normas, reglas y procedimientos de toma de decisiones que regulan y gobiernan un espacio transnacional, y que permite converger (de ahí la noción de integración) los principios, normas, reglas y procedimientos que operan entre sectores y a través de fronteras subnacionales, nacionales y regionales. El concepto es elaborado en Bruszt y McDermott (2009). 
contradictorias. En efecto, incluso la formación de nuevos Estados-naciones con capacidades mejoradas para una regulación sofisticada y para la integración social, que ha sido la agenda de muchos países de Europa del Este después del desplome del socialismo, parece ser compatible con las metas de las economías del centro, al menos en el contexto de una UE ampliada.

Esto nos conduce a evaluar los cambios en los factores internos del desarrollo, su diversidad e impacto variado, que había sido la segunda proposición clave de Cardoso y Faletto. En el caso de nuestras regiones, mientras el nuevo contexto global alteró el rango y el tipo de riesgos y oportunidades inherentes a diferentes alternativas de desarrollo, éstas han permanecido condicionales al "carácter de la organización social... dando considerable importancia a los efectos de la diversidad de recursos naturales, la locación geográfica... de cada economía" (Palma, 1978: 909-910). La importancia de los factores internos puede ser evaluada sobre dos dimensiones.

En primer lugar, los países post-socialistas y los países latinoamericanos no han tenido uniformemente buenas oportunidades de capitalizar la reubicación de industrias manufactureras complejas y/o la creciente voluntad de los súperpoderes regionales y globales para integrar sus "patios traseros". Más bien, ambas regiones contaron con impulsos externos para aumentar sus potenciales únicamente en la medida en que dichos súperpoderes estuvieron preparados y deseosos de hacerlo.

Es importante destacar que ambos, el sistema socialista y el capitalismo latinoamericano, incluyeron economías con niveles de desarrollo sorprendentemente variados. Los complejos industriales transnacionales, sin embargo, son raramente construidos de la nada. Se puede argumentar que los países que se especializaron durante el socialismo tardío en Europa del Este o que adquirieron "experiencia manufacturera" en fases sucesivas de la industrialización por sustitución de importaciones (ISI) en América Latina, son más proclives a recibir abundantes flujos de inversión extranjera directa (IED) que países en los cuales tales legados faltaron (Greskovits, 2005; Amsden, 2001).

Una diferenciación similar puede ser observada respecto al desarrollo social en general. Al comparar la "modernidad" de los países del ECE con aquella de los tigres asiáticos del tercer nivel -tales como Tailandia o Malasia-, Commisso afirma que los "indicadores sociales de modernización -urbanización, mortalidad infantil, tasas de alfabetismo y expectativa de vida- permanecen más altos en Europa del Este (...) y reflejan diferencias entre economías en vías de industrialización y países industrializados" (1998; 3, 4). A un nivel generalmente más bajo, los países latinoamericanos exhiben disparidades similares de desarrollo social. La diversidad de circunstancias internas tanto económicas como sociales indica que las situaciones concretas de dependencia son compatibles con un espacio ampliamente desigual para los agentes del desarrollo en los países del este y del sur.

En segundo lugar -como se demostrará más adelante- las grandes diferencias en la integración social alcanzada, mantenida (o incluso ampliada) por medio de políticas públicas de bienestar, no han sido homogeneizadas por las nuevas formas de inserción en la economía 
global. Más bien, grandes diferencias permanecen en esta dimensión tanto entre como en los países del este y del sur, poniendo en cuestión los supuestos pesimistas de una "carrera hacia el piso" (race to the bottom) respecto a salarios, empleo y, especialmente, prestaciones de bienestar social. Sorprendentemente, la creciente inserción global y la integración/inclusión social no aparecen contradictorias per se. Una trayectoria de desarrollo dependiente con un "rostro humano" parece ser aún posible.

Esta observación nos ayuda a evaluar la tercera y más importante proposición del paradigma del desarrollo dependiente: es exclusivamente a través de su articulación en la competencia política y el conflicto que pueden ser completamente capturados la interacción y el impacto sobre el desarrollo de los factores internacionales e internos. Sin embargo, el resultado de tales luchas es abierto, especialmente en un contexto democrático. Consecuentemente, Cardoso y Faletto -posibilistas moderados en el sentido de Hirschman (1988)- rechazaron las profecías fatalistas, las cuales vaticinaban posiciones predeterminadas similares para todos los países del sur en el sistema-mundo capitalista. En cambio, los autores de Dependencia y desarrollo argumentaron que la política era fundamental para los resultados del desarrollo; en nuestra perspectiva, hay mucho que aprender de su aproximación a este respecto también. En este ámbito mencionaremos algunos de los contextos contemporáneos en los cuales la política interna parece jugar un rol crucial.

En primer lugar, la advertencia de Cardoso y Faletto suena tan convincente hoy, en la era global, como antes: el desarrollo en países menos desarrollados es posible, pero es improbable a menos que esté enraizada en coaliciones desarrollistas en las cuales participen el capital transnacional y nacional, el Estado, y los trabajadores (para una elaboración pionera, véase Evans, 1979, y para una primera adaptación a los países post-socialistas de Europa del Este, véase Greskovits, 2003). Específicamente, las capacidades de reestructuración y desarrollo de las empresas nacionales resultan malogradas si dichas empresas no cuentan con transferencias de tecnología extranjera, capital y prácticas de marketing y administración avanzadas. Del mismo modo, la ausencia de aliados entre el empresariado nacional puede socavar las oportunidades de las empresas transnacionales de asegurarse recursos naturales locales, trabajo y emprendimiento con el fin de su propia reestructuración y expansión. Ambos tipos de empresas (local y transnacional), sin embargo, son dependientes de la capacidad que tenga la fuerza de trabajo de adaptarse a nuevas condiciones de competencia y de cambio estructural.

Además, capital y trabajo dependen, igualmente, del Estado como proveedor de bienes públicos, tales como entornos legales y macroeconómicos favorables, estabilidad política, infraestructura, regulación específica, e incentivos (por ejemplo, protección a la industria naciente) y como un importante organizador de sus redes de cooperación. Por otra parte, los trabajadores dependen del Estado como moderador de desequilibrios sociales, sectoriales y regionales. Por último, pero no por ello menos importante, la participación en una coalición desarrollista puede ayudar a las burocracias a desarrollar las habilidades, seleccionar y monitorear las políticas, así como construir y afinar las instituciones que pueden ampliar las oportunidades para un desarrollo sostenible. 
Sin embargo, la emergencia de coaliciones desarrollistas es un proceso inherentemente conflictual. Implica una coordinación entre objetivos sociales que son ampliamente divergentes y habitualmente contradictorios. La cuestión política central es cómo distribuir los beneficios durante los tiempos buenos y las cargas durante los tiempos difíciles entre un grupo de participantes tan diverso. Traducido a programa político, se trata de una estrategia cercana a lo que Cardoso ha llamado "una social democracia global" (Cardoso, 2009).

En segundo lugar, las nuevas políticas de los RTI contribuyen a la complejidad de las tensiones políticas y las luchas ya mencionadas. No es sorprendente que la reubicación de empresas de Europa Occidental en países de Europa del Este y la inmigración de trabajadores de bajo costo y su impacto social hayan llegado a ser aspectos altamente politizados en el curso de la ampliación de la UE. Los líderes occidentales criticaron a las empresas transnacionales por su falta de patriotismo, y a los nuevos Estados miembros por un supuesto dumping social. En su visión, la reubicación de empresas y la inmigración de trabajadores implicaban un potencial socavamiento de las relaciones industriales y los estados de bienestar de Europa Occidental. Por su parte, los políticos de Europa del Este vieron con recelo las murallas de proteccionismo en la Europa unida e indicaron las implicaciones negativas para la libertad y solidaridad. Del mismo modo, han estallado debates políticos candentes acerca del impacto del NAFTA sobre la economía estadounidense.

Basándonos en el paradigma del desarrollo dependiente, hemos identificado un nuevo patrón de dependencia que se manifiesta en el liderazgo de las corporaciones transnacionales de países del centro en áreas como tecnología, administración y marketing, así como relaciones de poder asimétricas en los nuevos RTI. Se ha argumentado, asimismo, que las nuevas manifestaciones de la dependencia no debieran ser consideradas más como limitantes de una (re)industrialización de manufacturas complejas, de la construcción de capacidades estatales, o de alcanzar integración social doméstica, al menos en un puñado de países de desarrollo tardío. En lo que sigue analizaremos cómo se comportan nuestras hipótesis al ser contrastadas con la evidencia empírica del desarrollo en Europa del Este y América Latina a principios de los años 2000.

\section{INTEGRACIÓN INTERNACIONAL PROFUNDA VERSUS SUPERFICIAL Y ESPECIALIZACIÓN SEMICENTRAL, SEMIPERIFÉRICA Y PERIFÉRICA}

En primer lugar discutiremos la dimensión correspondiente a los modos de integración internacional. Durante la década de 1990 los países del ECE se integraron rápidamente tanto a la economía europea como a la economía global. El valor de su comercio exterior se aproximó o excedió al de su Producto Interno Bruto (PIB). A través de sustanciales flujos de inversión extranjera directa, sus activos fueron incorporados a los sistemas de producción, finanzas y comercio tanto europeos como globales. A inicios de los 2000 el control extranjero ya es la norma en todas las mayores industrias y servicios del sector exportador. En el sector bancario, por su parte, la penetración extranjera ha alcanzado niveles récords prácticamente sin precedentes en otras partes de Europa y el mundo. Dicha exposición a influencias externas ha sido exacerbada fuertemente con la entrada al Fondo Monetario Internacional, 
al Banco Mundial, la Organización para la Cooperación y el Desarrollo Económico (OCDE), la Organización Mundial de Comercio (OMC) y, luego de una larga preparación, la UE. En resumidas cuentas, la internacionalización y la transnacionalización de los países del ECE ocurrió vía múltiples canales fuertemente institucionalizados, arraigando los procesos de cambio institucional en un RTI más complejo y profundo (Bohle y Greskovits, 2007b: 93, 94; Bruszt y Holzacker, 2009; acerca de la comparación de RTIs, véase Bruszt y McDermott, 2009).

Muy diversamente, la mayoría de los países de la Comunidad de Estados Independientes $(\mathrm{CEI})^{2}$ y los Balcanes Occidentales aún tienen que adquirir su membresía en la OMC, y su entrada a la UE y OCDE se encuentra más allá de sus posibilidades. Estos países han sido más cautelosos en liberalizar el comercio y los flujos de IED, manteniendo la propiedad nacional del sector bancario mucho más amplia que la de los países del ECE. A diferencia de la integración regional y global múltiple que ha caracterizado a los países del ECE, los patrones de integración de los CEI y de los Balcanes Occidentales aparecen con un bajo nivel de institucionalización y más bien superficiales.

Los modos de integración, superficiales y profundos, implican diferentes regímenes de internacionalización del cambio institucional interno, es decir, implican diferentes combinaciones de mecanismos de transnacionalización. La integración superficial implica un proceso de transnacionalización que es primariamente basado en incentivos, sanciones positivas y negativas provenientes de mercados liberalizados, o en condicionalidades provenientes de una organización externa (la que puede contar con mecanismos de exigibilidad más o menos estrictos). La integración profunda implica un régimen transnacional más complejo en el cual los mercados y las jerarquías son complementados por densas redes horizontales transnacionales y formas variadas de involucramiento extranjero, que puede incluir programas de asistencia orientados a transformar las capacidades de los actores públicos y privados. Además, los modos de integración profunda incluyen formas múltiples de monitoreo y de coordinación que ayudan a negociar el cambio institucional doméstico y a ajustarlo a diversos contextos locales.

Los patrones de integración profunda y superficial se vinculan con segmentos y nichos del mercado mundial de muy variada índole. En conjunto con los diferentes niveles de penetración de IED, estos reflejan diversos perfiles concretos de inserción global, es decir nuestra segunda dimensión de capitalismo transnacional (véase Tabla 1). Muchos países del ECE se integraron a los circuitos europeos y globales de producción y comercio a través de complejas industrias manufactureras basadas en capital físico y humano intensivos. Dichos países exportan al oeste lo que el oeste normalmente exporta al resto del mundo: químicos, maquinaria y equipamiento.

2 N. del T.: Asociación de Estados compuesta por 10 de las 15 ex repúblicas de la Unión Soviética, creada en 1991 luego de su disolución. No pertenecen a ella los países bálticos, miembros de la UE, ni Georgia que se retiró a fines de los 2000. 
Sin embargo, exportar autos y equipamiento electrónico puede implicar grados diferentes de sofisticación y de capacidades en diferentes economías. A este respecto, los datos disponibles para los países del ECE muestran una imagen mixta y dinámica que indica la necesidad de investigaciones más profundas si se quiere arribar a un juicio sólido acerca de la relación entre especialización sectorial, nivel de desarrollo actual y perspectivas de desarrollo futuras. No obstante, a la luz de dichos datos, nos atrevemos a proponer que -parafraseando la terminología de Immanuel Wallerstein (1979), aunque sin compartir todos sus supuestos y predicciones- la mayoría de los países del ECE están adoptando aspectos de semicentro (acerca de los aspectos conceptuales y empíricos de los perfiles de semicentro, semiperiferia y periferia en Europa del Este, véase Greskovits, 2005). Mientras la competitividad que estos países han adquirido en industrias manufactureras complejas es indiscutible, ella ha ido inextricablemente asociada al liderazgo del capital extranjero. A pesar del progreso reciente en mejoramiento tecnológico, los roles productivos continúan siendo caracterizados por niveles más bajos de autonomía, sofisticación y capacidades en comparación con las actividades concentradas en los segmentos occidentales de la misma industria transnacional.

Tanto los países bálticos así como Bulgaria y Rumania -países que se encuentran en el nivel más bajo del espectro de exportaciones en el ECE- Ilegaron a ser destino de muchas maquilas de textiles, vestuario, calzado, muebles, alimentos y artículos electrónicos de baja sofisticación orientadas a la exportación al mercado de Europa Occidental. Estos países combinan dicha especialización con exportaciones en industrias pesadas de contenido tecnológico bajo a medio tales como hierro, acero y metales no ferrosos, sectores claves a su vez en países como Bielorrusia y Ucrania. Estas economías quedan fuera del estatus de semicentro, calzando mejor con el estatus de semiperiferia. Las economías bálticas también deben ser colocadas aparte debido a sus especificidades: dado que constituyen los enclaves más occidentales de la ex Unión Soviética ahora dentro de los límites de la UE, estos Estados todavía se ganan una buena parte de su vida gracias a los servicios relacionados con el tránsito comercial ruso de petróleo y otros recursos, así como de bienes importados desde Rusia.

Finalmente, la mayoría de los países de la CEI presentan un tercer patrón de integración global. En estos países, los productos industriales complejos corresponden solo al $10 \%$ de sus exportaciones, mientras petróleo, gas, metales, algodón, piedras preciosas y alimentos corresponden al grueso de sus exportaciones. Debido a ello, estas economías corresponden más bien a economías periféricas.

La Tabla 1 muestra algunas referencias comparativas regionales. El capitalismo transnacional del sur parece mostrar una diversidad similar a la de las economías de mercado post-socialistas; de hecho, los países latinoamericanos se han embarcado también en trayectorias de semicentro, semiperiferia y periferia. No obstante, existen claras diferencias en la frecuencia relativa de la especialización: en comparación con Europa del Este, las economías latinoamericanas ocupan mayoritariamente el extremo más bajo de la división internacional y transnacional del trabajo. 


\section{TABLA 1}

Penetración de IED y perfiles de integración comercial (2006)

\begin{tabular}{|c|c|c|c|}
\hline $\begin{array}{l}\text { PERFIL DE INTEGRACIÓN } \\
\text { COMERCIAL (EXPORTACIONES } \\
\text { COMPLEJAS COMO PORCENTAJE } \\
\text { DE LAS EXPORTACIONES TOTALES)/ } \\
\text { PENETRACIÓN DE IED (STOCK DE } \\
\text { IED COMO PORCENTAJE DEL PIB) }\end{array}$ & $\begin{array}{l}\text { SEMICENTRO: } \\
\text { 40\% O MÁS }\end{array}$ & $\begin{array}{c}\text { SEMIPERIFERIA: } \\
21-39 \% \\
\text { POR CIENTO }\end{array}$ & $\begin{array}{c}\text { PERIFERIA: } \\
\text { MENOS DE } 21 \%\end{array}$ \\
\hline Alta: $50 \%$ o más & $\begin{array}{l}\text { Hungría }(73, \underline{79}) \text {, } \\
\text { R. Checa }(55, \underline{61}) \text {, } \\
\text { Eslovaquia }(55, \underline{57}) \text {, } \\
\text { Croacia }(63, \underline{40})\end{array}$ & $\begin{array}{l}\text { Estonia }(77, \underline{38}), \\
\text { Bulgaria }(66, \underline{22})\end{array}$ & $\begin{array}{l}\text { Azerbaiyán }(67, \underline{7}) \text {, } \\
\text { Panamá }(75, \underline{6}) \text {, } \\
\text { Chile }(55, \underline{6}), \\
\text { Nicaragua }(51, \underline{4})\end{array}$ \\
\hline Media: 25-49\% & $\begin{array}{l}\text { Polonia }(31, \underline{50}) \text {, } \\
\text { Costa Rica }(32, \underline{51}) \text {, } \\
\text { México }(27, \underline{\mathbf{6 2}})\end{array}$ & $\begin{array}{l}\text { Georgia }(43, \underline{30}) \text {, } \\
\text { Letonia }(38, \underline{26}), \\
\text { Lituania }(37, \underline{35}), \\
\text { Rumania }(34, \underline{37}), \\
\text { Argentina }(\mathbf{2 7}, \underline{\mathbf{2 1}})\end{array}$ & $\begin{array}{l}\text { Kazajistán }(42, \underline{6}) \text {, } \\
\text { Moldavia }(40, \underline{11}) \text {, } \\
\text { Macedonia }(39, \underline{10}) \text {, } \\
\text { Armenia }(27, \underline{3}), \\
\text { Bolivia }(45, \underline{4}), \\
\text { Ecuador }(\mathbf{4 0}, \underline{5}), \\
\text { Colombia }(33, \underline{15}), \\
\text { Honduras }(32, \underline{11}), \\
\text { Venezuela }(25, \underline{\mathbf{2}})\end{array}$ \\
\hline Baja: menos de $25 \%$ & Eslovenia $(20, \underline{56})$ & $\begin{array}{l}\text { Ucrania }(21, \underline{24}) \text {, } \\
\text { Bielorrusia }(7, \underline{30}) \text {, } \\
\text { Brasil }(21, \underline{\mathbf{3 1}})\end{array}$ & $\begin{array}{l}\text { Tayikisáan }(23, \underline{3}), \\
\text { Kirguistán }(21, \underline{13}), \\
\text { Rusia }(20, \underline{8}), \\
\text { Albania }(15, \underline{5}), \\
\text { Turkmenistán }(14, \underline{7}) \text {, } \\
\text { Uzbekistán }(8, \underline{20}) \\
\text { El Salvador }(\mathbf{2 4 , 1 9}) \text {, } \\
\text { Uruguay }(\mathbf{2 3}, \underline{\mathbf{1 2}}) \text {, } \\
\text { Perú }(\mathbf{2 1}, \underline{\mathbf{3}}), \\
\text { Paraguay }(\mathbf{1 9}, \underline{\mathbf{4}}), \\
\text { Guatemala }(\mathbf{1 4}, \underline{\mathbf{1 5}})\end{array}$ \\
\hline
\end{tabular}

Fuente: Cálculo de los autores sobre la base del World Investment Report y Base de Datos UN Tradecom (World Investment Report, 2007: Transnational Corporations, Extractive Industries and Development United Nations Conference on Trade and Development, disponible en www.unctad.org/Templates/ WebFlyer.asp?intltemID=4361\&lang=1).

(En paréntesis: Exportaciones complejas sobre las exportaciones totales).

Aun cuando la dependencia de las inversiones extranjeras -sustancial aunque variada-es característica en ambas regiones, la penetración de IED ha dirigido sólo excepcionalmente los perfiles de producción y exportación semicéntrico y semiperiférico de América Latina. Esto se explica principalmente por las grandes diferencias en los patrones sectoriales de la 
IED. En América Latina (así como en muchos de los países de la CEI) hay una concentración generalmente más fuerte de IED en industrias manufactureras de baja especialización y bajas remuneraciones, y en industrias basadas en recursos naturales (véase Bohle y Greskovits, 2007a, para datos sobre la estructura sectorial de IED en países del ECE y la explicación de la diversidad adoptada en este ensayo).

Generalmente, ni los altos ni los bajos grados de penetración de IED per se aparecen como la causa o el remedio de una especialización global desfavorable; el dónde la IED se acumula pareciera ser más relevante. Sin embargo, cabe preguntarse, ¿puede la inversión extranjera por sí sola conducir a una inserción exitosa en la economía global más allá de su orientación sectorial? Y viceversa, ¿puede la amplia diversidad en la especialización económica decirnos algo importante acerca de la organización social y política interna, de las fortalezas y debilidades, y de las posibilidades futuras de alterar las oportunidades de desarrollo en el este y en el sur?

\section{CAPACIDADES TRANSFORMATIVAS DEL ESTADO VERSUS FALTA DE ESTADO EN EL SEMICENTRO, SEMIPERIFERIA Y PERIFERIA}

Tomando en cuenta la evidencia anterior, es importante preguntarse cuán lejos podrán los Estados post-socialistas de Europa del Este y los Estados capitalistas pobres de América Latina retener o desarrollar un cierto grado capacidades transformativas y desarrollistas, es decir, una capacidad de asistir "la economía, transformarse a sí mismo y de responder a los cambios del entorno económico global" (Stepan, 1988; Gilpin, 1987: 77, citado en Shafer, 1994: 11).

Nuestra tercera dimensión -acerca de las capacidades del Estado para regular la economía, controlar la corrupción y sostener los principios del derecho, medidas por los indicadores de la calidad de la gobernanza del Banco Mundial (Kaufmann et al., 2004)- pareciera estar íntimamente relacionada con la especialización económica (Tabla 2). Hemos testeado la utilidad de los datos del Banco Mundial correlacionándolos con datos del Banco Europeo para la Reconstrucción y Desarrollo (EBRD) disponibles para los países del ECE únicamente, que miden capacidades regulativas en mercados financieros y de capitales así como en políticas comerciales. Además, hemos controlado los indicadores del Banco Mundial utilizando datos regionales comparativos sobre regulaciones en contra de la violación de derechos laborales (Mosley y Uno, 2007). Tomando en cuenta la fuerte correlación con estas bases de datos alternativas, las mediciones del Banco Mundial nos parecen aproximaciones confiables para medir las capacidades económicas del Estado.

Al examinar las relaciones entre capacidades del Estado y especialización económica, una simple regla general parece surgir: si no existe una calidad de gobernanza alta (o al menos media), tampoco existe un perfil de semicentro. En efecto, la mayoría de los Estados que son menos capaces se han especializado en roles periféricos basados principalmente en exportaciones de materias primas. Ambos hallazgos parecen confirmar una de las proposiciones inspiradoras de la literatura sobre desarrollo tardío (Stallings, 1990; Shafer, 1994: Karl, 1997). Como ha señalado Chaudry, el impacto de los activos financieros "regulados exclusivamente por las fuerzas del mercado internacional... tales como los 


\section{TABLA 2}

Calidad de la gobernanza y perfil de integración comercial (2006)

\begin{tabular}{|c|c|c|c|}
\hline $\begin{array}{l}\text { PERFIL DE INTEGRACIÓN } \\
\text { COMERCIAL (EXPORTACIONES } \\
\text { COMPLEJAS COMO PORCENTAJE } \\
\text { DE LAS EXPORTACIONES TOTALES)/ } \\
\text { CALIDAD DE LA GOBERNANZA } \\
\text { (PORCENTAJES NORMALIZADOS) }\end{array}$ & $\begin{array}{l}\text { SEMICENTRO: } \\
\text { 40\% O MÁS }\end{array}$ & $\begin{array}{l}\text { SEMIPERIFERIA: } \\
21-39 \%\end{array}$ & $\begin{array}{c}\text { PERIFERIA: } \\
\text { MENOS DE } 21 \%\end{array}$ \\
\hline Alta: $50 \%$ o más & $\begin{array}{l}\text { Eslovenia }(68, \underline{56}), \\
\text { Hungría }(65, \underline{79}), \\
\text { R. Checa }(65, \underline{61}), \\
\text { Eslovaquia }(64, \underline{57}), \\
\text { Polonia }(58, \underline{50}), \\
\text { Croacia }(54, \underline{40}), \\
\text { Costa Rica }(\mathbf{5 8}, \underline{\mathbf{5 1}})\end{array}$ & $\begin{array}{l}\text { Estonia }(77, \underline{38}), \\
\text { Letonia }(63, \underline{26}), \\
\text { Lituania }(62, \underline{35}), \\
\text { Bulgaria }(52, \underline{22}), \\
\text { Rumania }(50, \underline{37})\end{array}$ & $\begin{array}{l}\text { Chile }(76, \underline{6}), \\
\text { Uruguay }(60, \underline{12}) \text {, } \\
\text { Panamá }(50, \underline{6})\end{array}$ \\
\hline Media: $40-49 \%$ & México $(49, \underline{62})$ & $\begin{array}{l}\text { Georgia }(43, \underline{30}) \\
\text { Brasil }(\mathbf{4 5}, \underline{\mathbf{3 1})} \\
\text { Argentina }(\mathbf{4 0 ,} \underline{\mathbf{2 1}})\end{array}$ & $\begin{array}{l}\text { Armenia }(45, \underline{3}), \\
\text { Kazajistán }(42, \underline{6}) \\
\text { El Salvador }(\mathbf{4 6}, \underline{\mathbf{1 9}}) \text {, } \\
\text { Colombia }(\mathbf{4 6}, \underline{15}), \\
\text { Perú }(\mathbf{4 3}, \mathbf{3})\end{array}$ \\
\hline Baja: menos de $40 \%$ & & $\begin{array}{l}\text { Ucrania }(38, \underline{24}), \\
\text { Bielorrusia }(29, \underline{30})\end{array}$ & $\begin{array}{l}\text { Moldavia }(38, \underline{11}), \\
\text { Rusia }(37, \underline{8}), \\
\text { Albania }(36, \underline{5}), \\
\text { Azerbaiyán }(35, \underline{7}), \\
\text { Kirguistán }(32, \underline{13}), \\
\text { Macedonia }(28, \underline{10}) \text {, } \\
\text { Tayikistán }(23, \underline{3}), \\
\text { Uzbekistán }(23, \underline{20}) \text {, } \\
\text { Turkmenistán }(14, \underline{7}) \\
\text { Guatemala }(38, \underline{15}) \text {, } \\
\text { Honduras }(37, \underline{11}), \\
\text { Nicaragua }(37, \underline{4}), \\
\text { Bolivia }(34, \underline{4}), \\
\text { Paraguay }(32, \underline{4}), \\
\text { Ecuador }(31, \underline{5}), \\
\text { Venezuela }(\mathbf{2 7}, \underline{2})\end{array}$ \\
\hline
\end{tabular}

Fuente: Cálculo de los autores sobre la base de Kaufmann et al. (2007) y Base de Datos UN Tradecom (Kaufmann, Daniel, Aart Kraay y Massimo Mastruzzi [2004]: "Governance Matters III: Governance Indicators for 1996, 1998, 2000, and 2002", The World Bank Economic Review, 18 (2), pp. 253-84; para la Base de Datos UN Tradecom véase http://comtrade.un.org).

(En paréntesis: Calidad de la gobernanza normalizada, en porcentajes) 
retornos provenientes del petróleo, las remesas de los trabajadores y las inversiones de cartera, difiere radicalmente del impacto que tiene la inversión extranjera, los préstamos internacionales... que favorecen un rol importante del Estado como negociador, administrador, y distribuidor de recursos" (1997: 25).

Los datos de la Tabla 2 son consistentes con nuestra diferenciación previa entre trayectorias de desarrollo profundas y superficiales en ambas regiones, y apoyan el argumento de Cardoso acerca del rol del Estado como creador y corrector de mercados (Cardoso, 2009). Un Estado capaz que en parte filtra y en parte ayuda a ajustar la economía nacional a las tendencias del mercado global y a sus shocks, es mucho más característico del primero que del segundo grupo de trayectorias.

Sin embargo, la Tabla 2 también revela diferencias importantes en y entre las regiones. En primer término, muchos Estados semiperiféricos de la Europa del Este (de manera especial los nuevos miembros de la UE) parecen ser tan capaces como los Estados semicentrales; en segundo lugar, la especialización periférica en América Latina en su conjunto parece ser consistente con una diversidad mayor en cuanto a calidad de gobernanza que aquella que se aprecia para el caso de Europa del Este. En efecto, algunos de los países latinoamericanos más y menos capaces -Chile y Uruguay por una parte, versus Ecuador y Venezuela, por la otra- son, bajo nuestras categorías, todos periféricos. ¿Podría quizás la diferencia en la dotación institucional inicial explicar la diversidad en el este y en el sur?

Tomando en consideración que la rápida transnacionalización económica de la mayoría de los países del ECE coincidió con la construcción de los nuevos Estados-naciones independientes, es bastante sorprendente que estos países presenten tan altos indicadores de gobernanza. Aparentemente, sus frágiles nuevas instituciones públicas no han sido cooptadas o debilitadas por el capital internacional. Con toda seguridad, un importante factor internacional que provee al menos parte de la explicación ha sido la incorporación a la UE, la cual ha permitido e incluso forzado a estos Estados a importar las habilidades e instituciones de la buena gobernanza (Bruszt, 2002).

A su vez, los casos latinoamericanos atípicos parecen apoyar los argumentos de Gereffi y Evans acerca de que en el ámbito de la producción transnacional "lo que cuenta no es tanto lo que tú produces, como qué rol juegas al producirlo" (Evans, 1995: 251, nota al pie 16; para un concepto elaborado sobre esta idea, véase Gereffi, 1995). En este sentido, las economías de Chile, Uruguay y Panamá pueden haber capturado segmentos del mercado mundial con niveles de valor agregado relativamente altos, aunque siguiendo su propio perfil de integración periférico.

¿Es la especialización global la única área en la cual el Estado tiene que recuperar un rol principal? Proponemos que la otra área fundamental en la cual la "estatalidad" (stateness) cuenta, es mantener o aumentar la cohesión de las sociedades nacionales así como limitar la desigualdad a través de la provisión de servicios públicos de bienestar. 
TABLA 3

Gasto público y desigualdad (2000/2001)

\begin{tabular}{|c|c|c|c|}
\hline $\begin{array}{l}\text { DESIGUALDAD (COEFICIENTE } \\
\text { DE GINI) / GASTO PÚBLICO } \\
\text { (PORCENTAJE DEL PIB) }\end{array}$ & $\begin{array}{c}\text { BAJA: } \\
\text { MENOS DE 35\% }\end{array}$ & $\begin{array}{l}\text { MEDIA: } \\
36-45 \%\end{array}$ & $\begin{array}{c}\text { ALTA: } \\
\text { SOBRE } 45 \%\end{array}$ \\
\hline Alto: sobre $40 \%$ & $\begin{array}{l}\text { Croacia }(51, \underline{29}), \\
\text { Eslovenia }(49, \underline{28}), \\
\text { Hungría }(48, \underline{27}), \\
\text { Bielorrusia }(47, \underline{30}), \\
\text { R. Checa }(45, \underline{25}), \\
\text { Eslovaquia }(44, \underline{26}), \\
\text { Polonia }(44, \underline{34}), \\
\text { Bulgaria }(38, \underline{29})\end{array}$ & & $\begin{array}{l}\text { Panamá }(51, \underline{56}) \\
\text { Costa Rica }(45, \underline{46}) \\
\text { Colombia }(41, \underline{59})\end{array}$ \\
\hline Medio: $31-40 \%$ & $\begin{array}{l}\text { Uzbekistán }(36, \underline{27}), \\
\text { Ucrania }(34, \underline{28}), \\
\text { Rumania }(33, \underline{31}), \\
\text { Albania }(31, \underline{28})\end{array}$ & $\begin{array}{l}\text { Macedonia }(40, \underline{39}) \text {, } \\
\text { Estonia }(35, \underline{36}), \\
\text { Latvia }(35, \underline{38}), \\
\text { Russia }(35, \underline{40}), \\
\text { Lithuania }(31, \underline{36}) \\
\text { Nicaragua }(\mathbf{3 4}, \underline{43}), \\
\text { Uruguay }(\mathbf{3 1}, \underline{45})\end{array}$ & Argentina $(35, \underline{53})$ \\
\hline Bajo: menos de $3 \%$ & $\begin{array}{l}\text { Moldavia }(29, \underline{33}), \\
\text { Kyrguistán }(26, \underline{30}), \\
\text { Kazajistán }(23, \underline{34}), \\
\text { Armenia }(21, \underline{34}), \\
\text { Azerbaiyán }(19, \underline{19}) \\
\text { Tayikistán }(18, \underline{33})\end{array}$ & $\begin{array}{l}\text { Turkmenistán }(21, \underline{41}), \\
\text { Georgia }(18, \underline{48}) \\
\text { Ecuador }(\mathbf{2 9}, \underline{\mathbf{4 4}}) \\
\text { Venezuela }(\mathbf{3 0 , 4 2})\end{array}$ & $\begin{array}{l}\text { Brasil }(30, \underline{58}), \\
\text { Bolivia }(30, \underline{60}), \\
\text { Chile }(23, \underline{57}), \\
\text { Paraguay }(19, \underline{58}) \text {, } \\
\text { Perú }(17, \underline{55}), \\
\text { Honduras }(16, \underline{54}) \text {, } \\
\text { México }(16, \underline{49}), \\
\text { Guatemala }(14, \underline{55}) \text {, } \\
\text { El Salvador }(14, \underline{52})\end{array}$ \\
\hline
\end{tabular}

Fuente: Cálculo de los autores sobre la base del World Development Report y CEPAL (World Development Report 2006: Equity and Development, descargado de http://go.worldbank.org/XP2234QDV0).

\section{INTEGRACIÓN SOCIAL DOMÉSTICA}

La relación entre las oportunidades en la economía global y la integración social, nuestra cuarta dimensión, es un tema en disputa. De acuerdo a los representantes del consenso neoliberal que dominó el debate sobre desarrollo a inicios de las reformas económicas en el este y el sur, para que la apertura tuviese efectos beneficiosos debía ir mano a mano con el desmantelamiento del rol social del Estado y la despolitización de los temas distributivos. Los representantes de la teoría del crecimiento económico endógeno, por otro lado, argumentaban que los países podían beneficiarse diversamente de la apertura dependiendo 
del nivel y características de la inversión en capital humano (Landa y Kapstein, 2001). Es decir, las políticas redistributivas orientadas a aliviar las desigualdades podrían tener efectos positivos. Al contrario, desmantelar la redistribución pública puede conducir a la desigualdad y el empobrecimiento frustrando el crecimiento $y / o$ socavando las oportunidades para una integración internacional favorable (Landa y Kapstein, 2001). Cardoso (2009) presenta un argumento similar cuando discute la estrategia de una "social democracia globalizada".

Con el fin de identificar patrones diferenciados de integración social, hemos agrupado los países del este y del sur de acuerdo al nivel de gasto social medido a través del gasto público general -a falta de indicadores de gasto en protección social más detallados que sean comparables-, y a su nivel de desigualdad medido por el coeficiente de Gini. Las sociedades con altos niveles de gasto social y bajos niveles de desigualdad las hemos considerado como mejor integradas, mientras que las sociedades que se desempeñan peor en ambas variables las hemos considerado pobremente integradas.

La diversidad encontrada en el capitalismo transnacional de los recién llegados resulta sorprendente a la luz de esta evidencia. En primer lugar, los datos del ECE indican en general una mayor capacidad y/o un compromiso con la integración social a través de la provisión de servicios de bienestar que contrastan con el caso de América Latina. Segundo, en el este, los países con perfiles de integración internacional de semicentro parecen ser compatibles con esfuerzos públicos costosos y relativamente efectivos orientados a la integración social: prácticamente la mitad de los Estados de los países del ECE con altos puntajes en la primera dimensión (gasto social) también tienen altos puntajes en la segunda (igualdad). Aparentemente, hasta el momento estos Estados no han sido forzados a elegir entre una integración global promisoria y la cohesión de sus sociedades. En consecuencia, no hay una clara evidencia en el este de una "carrera al piso" (race to the bottom). Muy por el contrario, el éxito en la competencia por IED sofisticada y por nichos de manufacturas complejas no ha requerido precarizar el Estado de bienestar, como argumentaba la agenda de desarrollo neoliberal (Bohle, 2008). El hecho de que ningún país haya logrado llegar al semicentro sin un grado importante de integración social parece avalar la teoría del desarrollo endógeno.

Tercero, los Estados de bienestar no son característicos de todo el este, ni exclusivos de los casos post-socialistas. Por un lado, el grueso de los Estados periféricos de la Europa del Este ha fracasado en el intento de estimular la integración social, al igual que muchas de sus contrapartes del sur. En efecto, en términos de esfuerzos de bienestar hay una considerable brecha incluso dentro de los nuevos miembros de la UE (Bohle y Greskovits 2007a). Mientras la UE ha jugado un rol importante en el desarrollo de las capacidades económicas transformativas de los Estados, su influencia no ha conducido a una convergencia en sus funciones sociales. Por otro lado, las variedades de capitalismo en Europa del Este y América Latina se superponen en términos de gasto público redistributivo. Al menos de modo marginal, las variedades de capitalismo de América Latina parecen incluir también una variante más pro bienestar.

Cuarto, las dos regiones divergen radicalmente en una variable clave de desempeño: desigualdad de ingresos. En efecto, la desigualdad es mucho mayor en América Latina incluso 
en los casos donde los esfuerzos para mitigarla a través del gasto público han sido comparables a los estándares del ECE. Esto puede ser, en nuestra opinión, parcialmente explicado por las grandes diferencias en los niveles iniciales de desigualdad. En la coyuntura crítica de la apertura de sus economías al mercado global, los reformadores de las sociedades postsocialistas igualitarias han enfrentado restricciones muy diferentes a aquellas enfrentadas por la ya desigual América Latina, donde los diversos grupos sociales ya habían experimentado oportunidades desiguales para hacer efectivas sus demandas al Estado (Karl, 2006, 2008).

Finalmente, mientras la desigualdad parece estar inversamente relacionada con la fortaleza de los esfuerzos públicos para reducirla en los países del ECE, esta relación es menos evidente para el caso de América Latina, donde en términos de redistribución tanto los países líderes como los rezagados aparecen igualmente colmados de disparidades sociales. Esto despierta dudas acerca de la eficiencia de los servicios públicos de bienestar (para un análisis de las políticas sociales en América Latina durante la década de 1990, véase Stallings y Peres, 2000, especialmente el capítulo 5; para un análisis comparado de las reformas de bienestar en América Latina, el Este Asiático y países del ECE, véase Haggard y Kaufman, 2008).

\section{FORMAS DE CAPITALISMO TRANSNACIONAL EN EVOLUCIÓN: UN MAPA DE GRUPOS POSIBLES}

Hemos identificado arriba, como dimensiones interrelacionadas de diversos capitalismos transnacionales, los modos de integración internacional, los perfiles de inserción global y los patrones de capacidad estatal e integración social. Cada una de estas cuatro dimensiones puede tener un valor más posibilitador o limitante desde la perspectiva de la distribución de roles y oportunidades más o menos ventajosas en la economía global.

En primer lugar, hemos considerado los modos de integración internacional, es decir, si acaso la internacionalización económica ha ocurrido vía múltiples canales altamente institucionalizados y conducentes al establecimiento de marcos regulativos transnacionales exigibles, o bien únicamente a través de mercados globales de recursos y finanzas. Este es un aspecto nuevo y aún poco estudiado del desarrollo transnacional del capitalismo; mientras ciertas formas de integración internacional más profundas, que combinan el involucramiento directo de actores supranacionales en los procesos domésticos de construcción de instituciones, pueden mejorar las capacidades de actores domésticos estatales y no estatales, otras formas de integración superficiales pueden conservar el statu quo institucional e incluso debilitar a los agentes de desarrollo nacionales (Bruszt, 2002; Bohle y Greskovits, 2007b: 93, 94; Bruszt y McDermott, 2009).

En segundo lugar, hemos observado las diferencias en el rol jugado en la división internacional del trabajo y en el nivel de penetración de IED como dimensiones de distintas situaciones de desarrollo dependiente. Asimismo, hemos indagado brevemente en las diferentes formas de desarrollo y dependencia que pudieran estar asociadas con dichas combinaciones.

Finalmente, moviéndonos desde las dimensiones externas a las internas, hemos considerado la dimensión de capacidades estatales y los patrones de integración social. En general, y 
muy en línea con el trabajo de Cardoso y Faletto, sostenemos que la inserción en el mercado mundial a partir de una base exportadora más diversificada y compleja, con un Estado más capaz y con una integración social más fuerte, ofrece un mayor margen para los agentes de desarrollo nacionales (véase también Cardoso, 2009).

También hemos encontrado que, aun cuando la posibilidad de combinación entre estas dimensiones es alta, el número de patrones realmente existente es limitado. De ahí la siguiente proposición: estas combinaciones representan distintas situaciones de desarrollo dependiente y formas divergentes de capitalismo transnacional. Aquí sólo podemos indagar en algunos patrones dejando su discusión en profundidad para investigaciones futuras.

En un espacio de propiedades imaginario de cuatro dimensiones que combine aspectos externos e internos, limitantes y posibilitadores de agentes de desarrollo, alrededor de la mitad de los países del ECE caerían más cerca del polo posibilitador y más lejos del polo limitante (Tabla 4). Las incrustaciones institucionales internacionales más completas de estas economías, su especialización semicéntrica y la profunda penetración de IED, combinadas con capacidades estatales relativamente altas e integración social más bien extendida, las coloca en una trayectoria de desarrollo que difiere de otros países en ambas regiones, no sólo en grado sino también en forma. Estas economías pudieran representar una versión de la "social democracia global" de Cardoso en el este.

Los países en el primer grupo enfrentan restricciones distintas a las de aquellos en el segundo grupo, incluido el resto de los nuevos países miembros de la UE. La situación de los países bálticos, Bulgaria y Rumania se caracteriza por una combinación similar de factores habilitantes y restrictivos sobre las dimensiones externas e internas. Una integración internacional profunda y capacidades estatales relativamente altas los separan de los casos restantes, aunque estas ventajas se combinan con capacidades y desempeños externos semiperiféricos e integración social débil.

Tercero, al interior de Europa del Este, Rusia y las repúblicas de Asia Central caen más cerca del polo donde la combinación de factores externos e internos parece menoscabar el desarrollo. En esta esquina, la institucionalización internacional superficial y el perfil periférico en los mercados mundiales se combinan con capacidades estatales débiles para asistir al desarrollo económico y mantener la cohesión social.

Cuarto, desde la perspectiva de un posibilismo moderado, una situación interesante de desarrollo dependiente caracteriza a aquellas ex-repúblicas de la Unión Soviética donde recientemente han explotado "revoluciones electorales", tales como Ucrania y Georgia (Bunce y Wolchik, 2006). Aun cuando comenzaron a alejarse de aquellas esquinas donde los elementos negativos van de la mano, su posición y dinámica son ambiguas tanto en las dimensiones externas como domésticas. Aun cuando fuera de la UE, la posibilidad de ser consideradas para membresía ha jugado un papel en su compromiso con reformas políticas y económicas radicales que pudieran, en caso de ser exitosas, proveer ejemplos para estrategias de emprendimiento democrático. En otras palabras, usando la democracia (y la promesa de membresía en la UE), estos países pudieran liberar al Estado de la sujeción 


\section{TABLA 4}

Formas evolutivas del capitalismo transnacional

Un mapa de combinaciones típicas de factores habilitantes y restrictivos

\begin{tabular}{|l|c|c|c|c|c|c|}
\hline $\begin{array}{l}\text { Modo de } \\
\text { integración } \\
\text { transnacional }\end{array}$ & + & + & - & $?$ & - & - \\
\hline $\begin{array}{l}\text { Perfiles } \\
\text { concretos } \\
\text { de inserción } \\
\text { global }\end{array}$ & + & - & - & - & - & $?$ \\
\hline $\begin{array}{l}\text { Patrones de } \\
\text { capacidad } \\
\text { estatal }\end{array}$ & + & + & - & $?$ & + & $?$ \\
\hline $\begin{array}{l}\text { Patrones de } \\
\text { integración } \\
\text { social }\end{array}$ & + & - & - & - & - & - \\
\hline Ejemplos & $\begin{array}{l}\text { ECE países } \\
\text { del semi- } \\
\text { centro }\end{array}$ & $\begin{array}{l}\text { Países } \\
\text { bálticos, } \\
\text { Rumania, } \\
\text { Bulgaria }\end{array}$ & $\begin{array}{l}\text { Rusia, repúblicas } \\
\text { del Asia Central, } \\
\text { periferia } \\
\text { latinoamericana }\end{array}$ & $\begin{array}{l}\text { Variantes de } \\
\text { emprendimiento } \\
\text { democrático }\end{array}$ & $\begin{array}{l}\text { Chile, } \\
\text { Uruguay }\end{array}$ & $\begin{array}{l}\text { Brasil, } \\
\text { Argentina }\end{array}$ \\
\hline
\end{tabular}

+ significa que los factores externos o internos son más posibilitadores que limitantes.

- significa que los factores externos o internos son más limitantes que posibilitadores.

? significa que los factores externos e internos son ambiguos.

a grupos oligárquicos y de ese modo mejorar la calidad de la gobernanza económica. Todo esto pudiera facilitar un cambio hacia trayectorias de desarrollo más promisorias a través de una mejor integración social.

Tal como lo aclara el ensayo de Cardoso, varios países latinoamericanos han caminado por senderos similares - excepto el hecho de que los incentivos y asistencia en el emprendimiento democrático de un "gran hermano" benigno parecen haber sido más escasos en el patio trasero de los Estados Unidos que en el de la UE (Cardoso, 2009)-. Los ejemplos de Chile y Uruguay, y hasta cierto grado Brasil, demuestran que el emprendimiento democrático es una alternativa de desarrollo plausible. Chile y Uruguay continúan bloqueados principalmente por los bajos niveles de diversificación económica, Brasil y Argentina por capacidades estatales relativamente débiles, mientras que la cohesión social es un problema en todos ellos.

Por su parte, si bien Costa Rica y México muestran un patrón de inserción en la división global del trabajo similar al de los países del ECE, se encuentran alejados por otra 
configuración de límites y oportunidades. Careciendo de incrustaciones internacionales altamente institucionalizadas, ambos países deben movilizar recursos políticos y sociales adicionales para utilizar las posibilidades de su inserción global ventajosa y así atacar sus problemas y tensiones particulares: Costa Rica aun con una integración social insuficiente y México con una capacidad estatal más bien débil, así como con altas disparidades sociales y regionales. Finalmente, nos inclinamos a posicionar al resto de países latinoamericanos en el grupo poblado por Rusia y los Estados de Asia Central y el Cáucaso.

\section{RASTREANDO LOS ORÍGENES DE LA DIVERSIDAD}

Nos permitimos ofrecer algunas proposiciones tentativas acerca de los factores que en algunos casos abren nuevas posibilidades para los agentes de desarrollo y en otros las cierran en círculos viciosos y en trampas del desarrollo.

Comencemos con los legados de los modelos estatistas previos. Parecido a lo que ocurre en América Latina, la diversidad en el desarrollo económico no es un rasgo nuevo en Europa del Este. Incluso antes de la transformación política en 1989 los países en esta región presentaban grandes diferencias en sus patrones de industrialización, el grado de distorsión industrial y la estructura del comercio exterior. Algunos de los ex países socialistas permanecieron autárquicos mientras que otros abrieron gradualmente sus economías al mercado mundial, y de manera similar al modelo ISI en América Latina, las estrategias de desarrollo comunistas también diferían en sus grados de estatismo pues algunos países experimentaron con reformas de mercado mientras que otros no (para las variaciones en la ISI, véase Hirschman, 1968, 1995; y Gereffi y Wyman, 2000).

Del mismo modo, luego de su caída los regímenes socialistas dejaron legados ampliamente diversos tanto en términos de estructuras económicas como instituciones. En la periferia occidental del ex bloque soviético, algunos países y repúblicas soviéticas tenían estructuras industriales relativamente diversificadas con capital físico y recursos humanos tecnológicamente sofisticados. Otros eran más vulnerables a la apertura del mercado y tenían recursos institucionales y humanos menos desarrollados para hacer frente a los desafíos. Dichas diferencias en legados económicos dieron forma al espacio que tuvieron los reformadores para establecer diversas alianzas desarrollistas y también han jugado un rol en el timing y el carácter del ingreso de IED.

Entre los legados, en segundo lugar, también consideramos la diversidad en los tipos de organización política. Como en el caso de América Latina, en los últimos años de socialismo la oposición política y la represión fueron variables en Europa del Este, lo que incidió en la manera en que estos regímenes se disolvieron y la diversidad de instituciones políticas y dinámicas Estado-sociedad que dejaron atrás (Stark y Bruszt, 1998). Aun cuando diversa en términos de forma y fuerza, la oposición política estuvo presente en la mayoría de los países del ECE y ha jugado un rol central en la institucionalización del pluralismo político en el periodo de cambio de régimen. En cambio, en muchas de las ex repúblicas soviéticas la virtual ausencia de oposición llevó a una transición de un tipo de régimen autocrático a otro. En países como Rusia y Ucrania, las oposiciones manifiestas fueron reprimidas y 
debilitadas en la última parte de los años ochenta, dejando espacio a la emergencia de regímenes con una fuerte concentración del poder político.

Estas diferencias han jugado un papel crucial en la naturaleza de las nuevas instituciones políticas, determinando a su vez el rango de actores e intereses relevantes, y quienes ganaron representación efectiva cuando comenzaron las reformas de mercado y se establecieron las reglas de la nueva economía privatizada (Bruszt, 2001, 2002). Podemos encontrar una fuerte correlación entre la presencia de pluralismo político al momento de la coyuntura crítica a inicios de los años noventa y las situaciones de desarrollo a inicios de los 2000. Hacia finales de los años noventa y los 2000 tenían capacidades estatales fuertes sólo aquellos países cuyos mecanismos de rendición de cuentas forzaron a las autoridades a tomar en consideración diversos intereses societales durante las reformas (Hellman, 1998). Pluralismo político, inserción diversificada en los mercados globales, y niveles de integración social relativamente mayores, van de la mano en Europa del Este. Las predicciones acerca de una elección forzada entre democratización y construcción de mercado exitosa resultaron erradas: en Europa del Este, la ausencia de pluralismo político o un pluralismo débil coincidió con la des-diversificación económica, una inserción internacional (semi)periférica y bajos niveles de integración social.

Las predicciones erradas acerca de los efectos negativos de la extensión de los derechos democráticos sobre el éxito de los nuevos mercados estaban basadas en dos supuestos interconectados. El primero era que una vez liberalizados, los mercados producirían los incentivos económicos necesarios para generar las instituciones económicas adecuadas. La segunda suposición sostenía que la politización de la transición podía amenazar tanto la liberalización de los mercados como el surgimiento de las instituciones adecuadas. Debido a su estructura social plana y desarticulada, versaba el argumento, la transformación económica crearía demasiados perdedores y un crecimiento muy acelerado de las desigualdades; los perdedores no tolerarían estos cambios y usarían sus recientemente adquiridos derechos políticos para frenar el proceso (para una crítica a este enfoque, véase Hellman, 1998).

Por el contrario, el miedo a los perdedores de las reformas de mercado resultó ser exagerado, mientras que los peligros representados por los ganadores iniciales de las liberalizaciones fueron subestimados. Estos últimos tenían fuertes incentivos para establecer las reglas de la economía privatizada en su favor, y como resultado de la privatización rápida, habían concentrado el poder económico. En países donde los Estados no institucionalizaron defensas bajo la forma de controles y balances, y donde el pluralismo político era débil, los ganadores tempranos pudieron capturar el Estado y utilizarlo para redistribuir riqueza y oportunidades entre ellos mismos. Por el contrario, la competencia política y la presencia de mecanismos de extensión de la rendición de cuentas han ayudado a fortalecer la capacidad del Estado de introducir normas públicas, que reflejan intercambios complejos y acomodos de intereses diversos a la economía privada (Hellman, 1998; Bruszt, 2002, 2006).

No podemos, sin embargo, encontrar esta fuerte correlación entre democracia y dimensiones del desarrollo en América Latina. Creemos que parte de las razones de las diferencias entre ambas regiones pudieran deberse a diferencias en los legados de los modelos 
de desarrollo previos. Los regímenes socialistas de Europa del Este dejaron sociedades con niveles relativamente bajos de desigualdad y economías con baja concentración del poder económico privado. Cuando a principios de los años noventa las reformas de mercado crearon economías privatizadas con poder económico concentrado, la presencia o ausencia de pluralismo político pudo colocar a estos países en trayectorias divergentes.

En América Latina, las diversas ISI dejaron atrás sociedades extremadamente desiguales con poder económico altamente concentrado. Aquí, las reformas neoliberales de los años ochenta e inicios de los noventa han disminuido aún más las posibilidades de que los grupos sociales más débiles puedan ejercer demandas efectivas sobre el Estado (Collier y Handlin, 2005; Karl, 2006). Mientras que en Europa del Este la ausencia o debilidad de pluralismo político derivó en la captura del Estado por grupos oligárquicos, varias de las democracias latinoamericanas durante los años noventa han estado cerca de lo que algunos denominan "trampa de bajo equilibrio" (low equilibrium trap) (Bruszt y McDermott, 2009). Los beneficiarios potenciales de las mayores capacidades económicas del Estado o de políticas sociales más inclusivas carecieron de recursos y canales para captar la atención del Estado de manera sistemática (Collier y Handlin, 2005; Karl, 2006, 2008). Adicionalmente, grupos económicos ya consolidados no necesitaron corromper al Estado sino que pudieron aprovecharse de la mera perpetuación del statu quo (Schneider, 2004). En ausencia de demandas societales diversificadas, las autoridades han tenido pocos incentivos para experimentar con reformas más abarcativas. Aquí puede radicar la importancia del rebrote de la movilización democrática de la última década en América Latina mencionada por Cardoso (2009). Una mayor capacidad de distintos grupos domésticos para realizar demandas efectivas, combinada con políticas más inclusivas, proveen las bases para un camino alternativo, alejado de la (semi)periferia, que denominamos emprendimiento democrático.

Además de esta diferencia interregional, entre las democracias del ECE con puntos de partida similares también encontramos variaciones en las trayectorias de desarrollo. Estas diferencias, sostenemos, tienen mucho que ver con la forma como las políticas transformadoras manejaron los legados de los regímenes previos, percibiéndolos en algunos países como activos, y en otros como cargas (Bohle y Greskovits, 2007a). En tanto paradigma de política dominante (Hall, 1993) tanto en el este como en el sur durante los años noventa, el neoliberalismo sugería un alejamiento drástico de los legados previos por medio de una "destrucción creativa". Mientras que el lenguaje era homogéneo alrededor de todo el mundo, las ideas del paradigma de políticas dominante fueron traducidas de manera diferente, a veces incluso divergente, a los distintos lenguajes de la política interna.

Aquí enfatizamos el rol que jugaron las percepciones que los actores políticos tuvieron de estos legados en cuanto activos y/o cargas, y en la movilización de apoyos políticos para sus visiones, generando así nuevos clivajes y conflictos sociales y políticos gracias al agrupamiento de partes de los legados y sus sostenedores en diferentes "grupos internos" y "grupos externos", y estableciendo trayectorias de desarrollo en direcciones divergentes.

Tal como lo establecieron Bohle y Greskovits (2007a), en países como Estonia, Lituania y Letonia donde la reconstrucción de instituciones económicas coincidió con la reconstrucción 
de la nación y el Estado, los legados de la dominación soviética en activos económicos fueron vistos como amenazas. En estos países, las autoridades pudieron reunir apoyo para una política identitaria excluyente combinada con reformas radicales. En Eslovenia, donde la búsqueda de reformas democráticas y económicas precedió a la construcción nacional, y la independencia llegó con homogeneidad étnica, la política identitaria pudo convertirse en el marco para un régimen político inclusivo. De este modo, las estrategias de transformación económica graduales adoptadas en este país edificaron más sobre la reconstrucción que sobre la destrucción de los legados del modelo de desarrollo previo. En medio de estos dos casos extremos, países como Hungría, Polonia, República Checa y Eslovaquia, los Ilamados países Visegrad, no pudieron utilizar una política identitaria para privar del derecho de representación a amplias capas de la población, o para movilizar un apoyo prolongado a las políticas transformadoras. Bajo condiciones de competencia democrática, las autoridades en dichos países debieron buscar equilibrios entre avanzar en reformas económicas compensando a los grupos más críticos, mientras se intentaba silenciar a los restantes.

Cardoso (2009) desarrolla un argumento similar para América Latina. Los factores estructurales o las presiones externas pueden explicar sólo en parte las dinámicas políticas de las transformaciones económicas dado que estas influencias han interactuado con demandas por identidades culturales y nacionales, y han dado forma a políticas económicas en direcciones divergentes. Pero mientras en el caso de Bolivia y Ecuador, como describe Cardoso, las demandas de identidad cultural por parte de grupos previamente marginalizados han alejado a las políticas de las reformas neoliberales, la política identitaria en los países bálticos ha conducido hacia reformas de mercado más radicales.

Diferencias en la institucionalización de la política, distribución de recursos entre diferentes actores para el ejercicio efectivo de la voz, y finalmente, alternativas acerca de quién y qué cuenta en las políticas transformadoras, han colocado a estos países en trayectorias de desarrollo divergentes. Los efectos de estos factores internos fueron en parte reforzados y en parte modificados por diversas influencias internacionales, tales como las preferencias de ubicación de las corporaciones transnacionales y la emergencia de regímenes de integración transnacionales $(\mathrm{RTI})$.

Las preferencias de ubicación de las corporaciones transnacionales fueron moldeadas y moldearon las diversas condiciones domésticas y resultaron en círculos virtuosos o viciosos de acumulación de capital extranjero: reindustrialización en algunos países y desindustrialización en otros. Aquí utilizamos el ejemplo de la IED en industrias complejas que ha preferido a los países Visegrad, sobre todo por los incentivos provenientes de las instituciones de mercado, paquetes de subsidios especiales y las relaciones dinámicas entre los perfiles industriales heredados y los reestructurados (Bohle y Greskovits, 2007a). A través de la IED las industrias complejas de estos países ganaron acceso a factores de producción tangibles e intangibles esenciales, mejoraron sus actividades, y desarrollaron fortalezas competitivas incluso en el mercado más exigente de la UE.

Como resultado de las opciones de políticas iniciales y la consiguiente divergencia en la evolución de sus estructuras de oferta (por ejemplo, presencia o ausencia de capacidades de 
capital físico y humano sofisticadas), los países bálticos no pudieron competir con los países anteriores por IED compleja. Despojados de los medios para actualizarse y reestructurarse, los mismos sectores que han acelerado el desarrollo en los países Visegrad han perdido en los países bálticos la intensa competencia global, sus mercados, factores productivos, e influencia sobre las políticas. Mientras la entrada de IED manufacturera intensiva en capital y alta cualificación a las economías bálticas no ha sido importante, la acelerada migración de industrias livianas transnacionales de occidente los transformó en una de las muchas maquilas de la UE (Bohle y Greskovits, 2007a).

Finalmente, destacamos dos efectos de los RTI sobre el cambio institucional doméstico y las trayectorias de desarrollo. Primero, además de ofrecer incentivos de políticas ampliamente divergentes, los RTI difieren en la manera como traducen los paradigmas de políticas dominantes a nivel global a los diversos objetivos de política regionales, y dan forma a alternativas de políticas domésticas y al cambio institucional. En América Latina, RTI como el NAFTA o el Mercosur no quisieron o no pudieron alterar el paradigma de políticas neoliberal dominante. La principal forma por la que estos RTI afectaron el cambio institucional doméstico fue la incrustación de estos países en un entorno de mercado competitivo, dejando la transformación de las condiciones internas a los incentivos de mercado.

La manera en que la UE tradujo el paradigma neoliberal predominante en condicionalidades no negociables es única, y no ha sido igualada por otros regímenes de integración transnacionales. Además de medidas y políticas diseñadas para la liberalización del comercio, ésta se extiende a una variedad de dominios políticos, sociales y económicos. Se requirió a los países candidatos que no sólo incorporaran efectivamente la ya extensa legislación comunitaria a las legislaciones nacionales, sino, más importante aún, que la implementaran adecuadamente en la práctica a través de estructuras judiciales y administrativas apropiadas. Esto significó combinar la adopción de 80.000 páginas de estándares y regulaciones de la UE con la construcción de las condiciones institucionales necesarias para cumplir las demandas de la UE, detalladas en 31 capítulos o dominios de políticas que varían desde protección del consumidor a gobierno corporativo, desde regulación bancaria hasta políticas de ayuda estatal, y desde protección ambiental hasta compras públicas. Los documentos de condicionalidad de la UE han puesto en claro que además de la liberalización, la construcción de mercados supone desarrollar capacidades institucionales -rehaciendo las capacidades administrativas y regulatorias del Estado, y generando capacidades estatales para el desarrollo (Bruszt, 2002)-.

En segundo lugar, los RTI también difieren en la manera en que afectan la demanda y oferta por cambio institucional doméstico (Bruszt y McDermott, 2009). Mientras que la UE empodera diversos actores públicos y privados, no sólo a través de recursos sino especialmente mejorando su participación política y funcional en los esfuerzos de construcción de instituciones, el énfasis del NAFTA en los incentivos económicos provee presiones ascendentes (bottom up), demasiado débiles para alterar las propiedades de las regulaciones y del Estado regulador. El alcance limitado de las condicionalidades del NAFTA, enfocadas en la liberalización del comercio y honrando ampliamente el statu quo en materia de regulación doméstica (Duina, 2007), conserva la posición de los actores domésticos más 
fuertes. Al mismo tiempo, la liberalización del comercio incrusta las disputas domésticas por cambio institucional en un entorno de mercado competitivo, limitando el espacio a fuerzas que tratan de diversificar los intereses y las consideraciones a tomar en cuenta a la hora de diseñar las reglas de la economía.

Conectando condicionalidades políticas y económicas, y a través de un monitoreo estrecho de la adopción de derechos políticos y de las reglas de competencia política justa, la UE ha mantenido constante la voz doméstica, o la ha incrementado en algunos países como Eslovaquia y en casi todos los países del sudeste europeo, proveyendo al mismo tiempo una direccionalidad clara y sin ambigüedades a las presiones domésticas ascendentes bajo la forma de condicionalidades para el acceso. Asimismo, la cuasi simultaneidad entre la liberalización del comercio y la re-incrustación de los mercados domésticos en un marco regulativo amplio y monitoreado transnacionalmente, ha fortalecido la posición negociadora de los Estados domésticos vis-à-vis las empresas domésticas buscadoras de rentas o las corporaciones transnacionales. También ha mejorado las oportunidades políticas de diversos actores económicos más débiles. La re-regulación de las reglas de competencia y gobierno corporativo, cambios en las reglas de ayuda estatal y compras públicas, la modificación de estándares ambientales o de protección del consumidor, por nombrar sólo algunos pocos dominios, han transformado las propiedades de los campos regulativos, los ha transnacionalizado, y ha incrementado las oportunidades de los actores más débiles para realizar demandas legítimas sobre el mejoramiento de las instituciones, permitiéndoles también participar en el monitoreo o exigibilidad de las normas. Finalmente, mientras en América Latina los RTI tienen medios muy limitados para ayudar a mejorar el lado de oferta del cambio institucional doméstico, la UE ha generado un programa de asistencia diversificado y múltiple. Con un presupuesto total de cerca de 28 mil millones de euros, estos programas se han enfocado en la construcción de capacidades tanto dentro como fuera del Estado, y han incluido la participación directa de miles de expertos y policymakers de los Estados miembros de la UE.

\section{CONCLUSIONES}

Nos permitimos concluir con algunas reflexiones acerca de la naturaleza cambiante de la dependencia, que también destacan la relevancia actual de la búsqueda de combinaciones sistemáticas de factores limitantes y habilitantes del desarrollo.

1. Enraizada en la distribución inequitativa de recursos, roles y oportunidades, la dependencia continúa siendo un aspecto importante de la división internacional del trabajo, aunque su naturaleza ha cambiado. En primer lugar, las economías de desarrollo tardío de Europa del Este y América Latina todavía dependen de los países céntricos y las corporaciones transnacionales en materia de nueva tecnología y habilidades de emprendimiento globales. Segundo, un puñado de países del Este Europeo y México se han unido a la UE y al NAFTA respectivamente, desde posiciones asimétricas vis-à-vis los países avanzados que dominan estos RTI. 
2. En vez de dificultar la industrialización, las nuevas formas de dependencia parecen compatibles con estructuras e instituciones industriales avanzadas. A través de la reubicación productiva desde el centro, la nueva división global del trabajo permite a algunos de los países en desarrollo recientes transformarse en productores y exportadores importantes de bienes manufacturados complejos, que por su dependencia intensiva en capital físico y humano eran vistos hasta ahora como la fuente principal de ventajas comparativas para las economías avanzadas. De este modo, la dependencia deja de estar en contradicción incluso con formas bastante complejas de industrialización.

3. La dependencia de los órganos centrales de la EU no es perjudicial para el fortalecimiento de las capacidades de desarrollo de los Estados domésticos. Por el contrario, a través de condicionalidades, programas de asistencia, e incentivos a la creación de redes de cooperación transfronterizas, la UE promueve la construcción de Estado en sus nuevos miembros y en los países candidatos. En efecto, como lo demuestra el ejemplo europeo, las relaciones asimétricas entre los países del centro y aquellos que se alejan de él no ponen en conflicto la formación de nuevos Estados nacionales con la reconquista de mejores capacidades de regulación, o espacios políticos y económicos recientemente diseñados -la agenda principal de tantos países del este europeo luego del colapso del socialismo-.

4. Lejos de poner en movimiento una "carrera hacia el piso" (race to the bottom) uniforme, el nuevo patrón de dependencia parece permitir cierto margen para una coexistencia precaria y políticamente disputada entre el imperativo de competitividad global y variados niveles de integración social. Esto es, los países no pueden "comprar" automáticamente posiciones promisorias dentro de la división internacional del trabajo por medio de la mera reducción del gasto social y la exclusión de grandes porciones de su población. Así, la dependencia no necesariamente socava la inclusión social. Más bien, depende de la lucha democrática el establecer un balance entre los requerimientos de integración social que sea promisorio externamente y aceptable políticamente.

\section{BIBLIOGRAFÍA}

Amsden, Alice H. (2001): The Rise of the Rest. Challenges to the West from Late-Industrializing Economies, Oxford University Press, Oxford.

Bohle, Dorothee (2008): "Race to the Bottom? Transnational Companies and Reinforced Competition in the Enlarged European Union", en Bastiaan Van Apeldoorn, Jan Drahokoupil y Laura Horn (eds.): European Neoliberal Governance and Beyond, Palgrave Macmillan, Basingstoke, en prensa.

Bohle, Dorothee y Béla Greskovits (2007a): “Neoliberalism, Embedded Neoliberalism and Neocorporatism: Towards Transnational Capitalism in East-Central Europe", West European Politics, 30 (3), pp. 443-66.

(2007b): "The State, Internationalization, and Capitalist Diversity in Eastern Europe", Competition \& Change, 11 (2), pp. 89-115.

Bruszt, Lászlo (2001): "Heterarchies and Developmental Traps", Brazilian Journal of Political Economy, 2001/1, pp. 37-57. 
(2002): "Making Markets and Eastern Enlargement: Diverging Convergence?", West European Politics, 25 (2), pp. 121-40.

(2006): "Making Capitalism Compatible with Democracy - Tentative Reflections from the East", en Colin Crouch y Wolfgang Streck (eds.): The diversity of democracy - A tribute to Philippe C. Schmitter, Edward Elgar, Northampton.

Bruszt, Lászlo y Gerald McDermott (2007): “Transnational Integration Regimes as Development Programs", trabajo presentado en la reunión anual de la APSA, Chicago.

Bunce, Valerie J. y Sharon Wolchik (2006): "Favorable Conditions and Electoral Revolutions", Journal of Democracy, 17 (4), pp. 5-18.

Cardoso, Fernando H. y Enzo Faletto (1979 [1967]): Dependency and Development in Latin America, University of California Press, Berkeley/Los Angeles.

Chaudry, Kiren A. (1997): The Price of Wealth. Economies and Institutions in the Middle East, Cornell University Press, Ithaca/London.

Collier, Ruth Berins y Samuel P. Handlin (2005): "Shifting Interest Regimes of the Working Classes in Latin America", Working Paper, Institute of Industrial Relations, University of California, Berkeley.

Duina, Francesco (2007): The Social Construction of Free Trade: The European Union, NAFTA, and Mercosur, Princeton University Press, Princeton.

Evans, Peter (1979): Dependent development: the alliance of multinational, state and local capital in Brazil, Princeton University Press, Princeton.

(1995): Embedded Autonomy. States \& Industrial Transformation, Princeton University Press, Princeton.

Gereffi, Gary (1995): "Global Production Systems and Third World Development", en Barbara Stallings (ed.): Global Change, Regional Response, Cambridge University Press, Cambridge, pp. 100-42.

Gereffi, Gary y Donald L. Wyman (eds.) (2000): Manufacturing Miracles: Paths of Industrialization in Latin America and East Asia, Princeton University Press, Princeton.

Gilpin, Robert (1987): The Political Economy of International Relations, Princeton University Press, Princeton.

Greskovits, Béla (2005): "Leading sectors and the varieties of capitalism in Eastern Europe", Actes de Gerpisa, 39, pp. 113-28.

Greskovits, Béla y Hector Schamis (1999): “Democratic Capitalism and the State in Eastern Europe and Latin America", trabajo presentado en la reunión anual de la APSA, septiembre 2-5, Atlanta, Georgia, mimeo.

Gryzmala-Busse, Anna y Pauline Jones Luong (2002): "Reconceptualizing the state: lessons from postcommunism", Politics \& Society, 30 (4), pp. 529-54.

Haggard, Stephan y Robert R. Kaufman (2008): Recrafting Social Contracts: Welfare Reform in Latin America, East Asia and Central Europe, Cambridge University Press, Cambridge, en prensa.

Hall, Peter A. y David Soskice (eds.) (2001): Varieties of Capitalism, The Institutional Foundations of Comparative Advantage, Oxford University Press, Oxford.

Hellman, Joel (1998): "Winners Take All: The Politics of Partial Reform in Postcommunist Transitions", World Politics, 50 (2), pp. 203-234.

Hirschman, Albert O. (1968): "The Political Economy of Import-Substituting Industrialization in Latin America", The Quarterly Journal of Economics, 82 (1), pp. 1-32. 
(1988): A Bias for Hope: Essays on Development and Latin America, Yale University Press, New Haven.

(1995): "On the Political Economy of Latin American Development", en Albert O. Hirschman: A Propensity to Self-Subversion, Harvard University Press, Boston, pp. 154-88.

Karl, Terry L. (1997): The Paradox of Plenty. Oil Booms and Petro-States, University of California Press, Berkeley, Los Angeles/London.

(2006): "From Democracy to Democratization and Back", en Colin Crouch y Wolfgang Streck (eds.): A Diversity of Democracy: In Honor of Philippe C. Schmitter, Elsevier, London.

(2008): "The Vicious Cycle of Inequality in Latin America", en Susan Eva Eckstein y Timothy P. Wickham-Crowley (eds.): The Politics of Injustice in Latin America, University of California Press, Berkeley, en prensa.

Kaufmann, Daniel, Aart Kraay y Massimo Mastruzzi (2004): "Governance Matters III: Governance Indicators for 1996, 1998, 2000, and 2002", The World Bank Economic Review, 18 (2), pp. 253-84.

Mosley, Layna y Saika Uno (2007): "Racing to the Bottom or Climbing to the Top? Economic Globalization and Collective Labor Rights", Comparative Political Studies, 40 (8), pp. 923-948.

Palma, Gabriel (1978): "Dependency: A Formal Theory of Underdevelopment or a Methodology for the Analysis of Concrete Situations of Underdevelopment?", World Development, 6, pp. 881-924.

Przeworski, Adam (1991): Democracy and the Market: Political and Economic Reforms in Eastern Europe and Latin America, Cambridge University Press, Cambridge/New York.

Schneider, Ben Ross (2004): "Varieties of Semi-Articulated Capitalism in Latin America", trabajo presentado en la reunión anual de la APSA, Chicago, septiembre 2-5.

Shafer, Michael D. (1994): Winners and Losers. How Sectors Shape the Developmental Prospects of States, Cornell University Press, Ithaca/London.

Stallings, Barbara (1990): "The Role of Foreign Capital in Economic Development", en Gary Gereffi y Donald L. Wyman (eds.): Manufacturing Miracles. Paths of Industrialization in Latin America and East Asia, Princeton University Press, Princeton, pp. 55-89.

(1992): "International Influence on Economic Policy: Debt, Stabilization and Structural Reform", en Stephan Haggard y Robert R. Kaufman (eds.): The Politics of Economic Adjustment: International Constraints, Distributive Conflicts, and the State, Princeton University Press, Princeton, pp. 41-88.

Stallings, Barbara y Wilson Peres (2000): Growth, Employment, and Equity. The Impact of the Economic Reforms in Latin America and the Caribbean, United Nations Economic Commission for Latin American and the Caribbean, Brookings Institution Press, Washington D.C.

Stark, David y Lászlo Bruszt (1998): Postsocialist Pathways: Transforming Politics and Property in East Central Europe, Cambridge University Press, Cambridge.

Stepan, Alfred (1988): Rethinking Military Politics, Princeton University Press, Princeton.

Wallerstein, Immanuel (1979): The Capitalist World-Economy, Cambridge University Press/Editions de la Maison des Sciences de I'Homme, Cambridge. 